\title{
Electron-ion dynamics in laser-assisted desorption of hydrogen atoms from $\mathrm{H}-\mathrm{Si}(111)$ surface
}

\author{
Sergiy Bubin ${ }^{a)}$ and Kálmán Varga \\ Department of Physics and Astronomy, Vanderbilt University, Nashville, Tennessee 37235, USA
}

(Received 24 May 2011; accepted 4 August 2011; published online 20 September 2011)

\begin{abstract}
In the framework of real time real space time-dependent density functional theory we have studied the electron-ion dynamics of a hydrogen-terminated silicon surface H-Si(111) subjected to intense laser irradiation. Two surface fragments of different sizes have been used in the simulations. When the intensity and duration of the laser exceed certain levels (which depend on the wavelength) we observe the desorption of the hydrogen atoms, while the underlying silicon layer remains essentially undamaged. Upon further increase of the laser intensity, the chemical bonds between silicon atoms break as well. The results of the simulations suggest that with an appropriate choice of laser parameters it should be possible to remove the hydrogen layer from the $\mathrm{H}$-Si(111) surface in a matter of a few tens of femtoseconds. We have also observed that at high laser field intensities $(2-4 \mathrm{~V} / \AA$ in this work) the desorption occurs even when the laser frequency is smaller than the optical gap of the silicon surface fragments. Therefore, nonlinear phenomena must play an essential role in such desorption processes. (C) 2011 American Institute of Physics. [doi:10.1063/1.3638064]
\end{abstract}

\section{INTRODUCTION}

Surfaces and interfaces of semiconductors play a fundamental role in device applications. ${ }^{1}$ The hydrogen terminated Si surface has emerged as the ideal surface for the fabrication of functionalized surfaces. ${ }^{2,3}$ Passivation of silicon surfaces by chemisorption of hydrogen provides long-term protection against oxidation and adsorption of impurities. Hydrogen-passivated silicon has bulk-like surface reconstruction $^{4}$ and can be used as a starting layer for epitaxial growth or as a resist layer for nanolithographic techniques. Selective removal of hydrogen from these fully nonreactive surfaces by using the tip of a scanning tunneling microscope $(\mathrm{STM})^{5-8}$ or by laser irradiation, ${ }^{9}$ enables the patterning of reactive areas. Adsorption of appropriate atoms or molecules into patterned areas can be used to fabricate functionalized structures. $^{2,3}$

In this article, we will study the hydrogen desorption from a $\mathrm{Si}(111)$ surface by intense laser pulses. Bond scission by laser pulses is a convenient way to induce and control chemical reactions. ${ }^{10-13}$ Selective control of surface reactions is especially desired. ${ }^{14}$ Among the many surfaces explored by experiments, the hydrogen terminated Si surface is the most studied. ${ }^{9,15,16}$ The desorption of atoms from surfaces can be induced by electronic excitations directly by coupling between the laser field and the bond electrons or by indirect mechanisms where the excitation of the substrate is transferred to the desorption coordinates. ${ }^{7,17,18}$

$A b$ initio approaches have been very successful in describing the physical properties of hydrogenated $\mathrm{Si}$ surfaces. ${ }^{19-24}$ These studies have revealed the surface relaxation mechanisms, ${ }^{19,21}$ surface atom vibrational modes, ${ }^{20,22}$ phonon-phonon interactions, ${ }^{25}$ and other structural and energetic properties. ${ }^{23}$ Hydrogen desorption from silicon has also

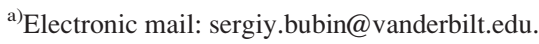

been subject to various theoretical studies. ${ }^{26-34}$ These studies mostly use reduced dimensionality quantum or quasiclassical dynamical simulations on density-functional-theory derived potential energy surfaces. ${ }^{33,34}$ First principles timedependent calculations ${ }^{35}$ have also been used to simulate the STM induced desorption: The Si-H bond of the hydrogenated S(111) surface was subjected to an excitation by promoting electrons from $\sigma$ to $\sigma^{*}$ orbitals and the motion of atoms was monitored in time after this excitation. In the present work we add a time dependent laser pulse to the Hamiltonian of the system and simulate the entire process on an equal footing.

In this article, we will carry out first-principles timedependent simulations of laser-assisted hydrogen desorption from a $\mathrm{Si}(111)$ surface using a real space real time density functional calculation complemented with Ehrenfest molecular dynamics. First principles simulations are indispensable tools to gain insight into the physical mechanisms behind the highly nonequilibrium and nonlinear laser induced desorption of hydrogen from the silicon surface. The results of the study enhance our understanding of the dynamics of electrons and atoms in and on surfaces. The present publication extends the studies presented in Ref. 36 and provides details of the numerical simulations.

Density functional theory (DFT) (Refs. 37 and 38) is now well established as one of the most capable methods for computing electronic ground state properties. In its original formulation, DFT only applies to the electronic ground state. Runge and Gross ${ }^{39}$ generalized DFT to time-dependent systems. According to the Runge-Gross theorem, for any given initial state of a many-electron system, a time-dependent potential acting on it is uniquely determined by the subsequent time evolution of the one-electron density. Using this theorem, it is possible to formally establish a time-dependent Kohn-Sham (TDKS) equation from which various oneparticle properties of the system can be obtained as functions 
of time. The resulting theoretical framework is usually referred to as time dependent density functional theory (TDDFT). ${ }^{40}$

There are two distinct flavors in which TDDFT calculations have been implemented. The first is based on direct time propagation of the wave function ${ }^{41-44}$ by solving the TDKS equations. The second approach uses an infinitesimal time dependent perturbation, then Fourier-transforms the equations into frequency space where the linear response function can be derived in closed form. ${ }^{45-47}$ In this work, the first approach will be used because we are interested in the dynamics of the process. To represent the Kohn-Sham (KS) orbitals the majority of TDDFT implementations use either atom-centered real space basis sets, ${ }^{48}$ which are common in quantum chemistry, ${ }^{49}$ or plane-wave expansions. ${ }^{50,51}$ Real space grids $^{42,52,53}$ are, however, also popular choices for representing the orbitals in TDDFT calculations. The main advantage of real space grids is that the convergence can be systematically controlled with a single parameter, the grid spacing. In the present work, real space grids have the additional advantage of being able to represent the diffuse electron cloud in the ionizing strong laser pulse.

In time propagation one has to calculate the action of the operator $\exp (-i H \Delta t / \hbar)$ on orbitals $\psi_{k}$, where $H$ is the Hamiltonian of the system and $\Delta t$ is the time step. Various techniques have been developed ${ }^{44,54-59}$ to approximate this operator including polynomial expansions of the exponential and the Crank-Nicholson ${ }^{60}$ approach. Once the Hamiltonian is represented as a matrix using appropriate basis states, time propagation involves repeated application of the Hamiltonian to calculate $H^{n}\left|\psi_{k}\right\rangle$ (polynomial approximation). A limitation of the time propagation based approach is that stable and accurate integration of the TDKS equations requires a time step as small as $10^{-3}$ fs in typical pseudopotential applications, and the time step usually decreases as the grid spacing increases.

TDDFT has been successfully used in various timedependent quantum mechanical simulations. The most important applications of the TDDFT approach are (i) nonperturbative calculations with systems in intense laser fields, ${ }^{41,61,62}$ (ii) calculations of optical response, dielectric functions, and electronic transitions, ${ }^{43,52,63-70}$ (iii) calculation of electronic excitations, ${ }^{71-73}$ and (iv) time-dependent transport calculations. ${ }^{74-76}$

The outline of the paper is as follows. After this introduction, the details of the computational method are presented in Sec. II. The numerical results are presented and discussed in Sec. III.

\section{METHOD}

In the framework of time-dependent density functional theory, ${ }^{39}$ the evolution of the system is determined by solving the time-dependent Kohn-Sham equations for singleparticle orbitals $\psi_{k}$,

$$
i \hbar \frac{\partial \psi_{k}(\mathbf{r}, t)}{\partial t}=H \psi_{k}(\mathbf{r}, t), \quad k=1, \ldots, N,
$$

where $H$ is the Kohn-Sham Hamiltonian given by

$$
\begin{aligned}
H= & -\frac{\hbar^{2}}{2 m} \nabla_{\mathbf{r}}^{2}+V^{\mathrm{ion}}(\mathbf{r},\{\mathbf{R}\})+V^{\mathrm{H}}[\rho](\mathbf{r}) \\
& +V^{\mathrm{XC}}[\rho](\mathbf{r})+V^{\mathrm{ext}}(\mathbf{r}, t) .
\end{aligned}
$$

Here $V^{\text {ion }}, V^{\mathrm{H}}$, and $V^{\mathrm{XC}}$ are the ionic core, Hartree, and exchange-correlation potentials, respectively. $V^{\text {ext }}$ is the explicitly time-dependent potential due to the electric field of the laser. $m$ stands for the electron mass, while $\mathbf{R}_{i}$ is the position of the $i$-th ion. The electron density, $\rho$ is computed as the sum over all occupied orbitals,

$$
\rho(\mathbf{r}, t)=\sum_{k=1}^{N} f_{k}\left|\psi_{k}(\mathbf{r}, t)\right|^{2}
$$

where $f_{k}$ are the occupation numbers. The simulations in the present work have been carried out using the adiabatic local density approximation with the parameterization of Perdew and Zunger ${ }^{77}$ for the exchange-correlation potential. To represent ionic core potentials we employed norm-conserving pseudopotentials in the form of Troullier and Martins. ${ }^{78} V^{\text {ion }}$ consists of a local and nonlocal part:

$$
\begin{aligned}
V^{\mathrm{ion}}(\mathbf{r},\{\mathbf{R}\})= & \sum_{i=1}^{N_{\text {ions }}}\left[V_{i}^{\mathrm{loc}}\left(\mathbf{r}-\mathbf{R}_{i}\right)\right. \\
& \left.+\sum_{l=1}^{L_{i}^{\max }} \sum_{m=-l}^{l} V_{i l m}^{\mathrm{nonl}}\left(\mathbf{r}-\mathbf{R}_{i}\right) \hat{P}_{l}\right],
\end{aligned}
$$

where operator $\hat{P}_{l}$ is a projector onto the subspace of angular momentum $l$. The Hartree potential is defined as

$$
V^{\mathrm{H}}[\rho](\mathbf{r}, t)=\int \frac{\rho\left(\mathbf{r}^{\prime}, t\right)}{\left|\mathbf{r}-\mathbf{r}^{\prime}\right|} d \mathbf{r}^{\prime}
$$

and was evaluated by numerically solving the Poisson equation. The last term in Eq. (2) representing the potential due to the laser can be written as

$$
V^{\mathrm{ext}}(\mathbf{r}, t)=x \mathcal{E}(t),
$$

which assumes the polarization of the oscillating electric field $\mathcal{E}(t)$ is along the $x$-axis. Since the shortest wavelength of the laser used in the present simulation (99 nm) significantly exceeds the size of the simulation box, the instantaneous amplitude of the laser can, to a good approximation, be considered independent of coordinates. Hence, the potential has a linear dependence on the $x$-coordinate only. The amplitude of the laser electric field was increased gradually from 0 to $\mathcal{E}_{\max }$ using the following Fermi-like ramping:

$$
\mathcal{E}(t)=\mathcal{E}_{\max }\left(1-\frac{1}{1+\exp [(t-a) / b]}\right) \sin \omega t,
$$

where $\omega$ is the frequency of the laser, while $a$ and $b$ are parameters which control the turn-on time and the smoothness of ramping. In our simulations we used $a=4.0$ fs and $b=0.75$ fs.

Initially, the system was put in its ground state by performing the ground state DFT calculation. After that each 
Kohn-Sham orbital, $\psi_{k}$, was time-propagated for a period of 25-30 fs. The propagation was achieved by a sequence of applications of the evolution operator corresponding to a small time step $\Delta t=0.001 \mathrm{fs}$, so that the Hamiltonian at time $t$ remains nearly commutative with the Hamiltonian at time $t+\Delta t$. The fourth order Taylor expansion was used to approximate the exponential form of the time evolution operator, $U(t, t+\Delta t)$ :

$$
\psi_{k}(\mathbf{r}, t+\Delta t) \approx \sum_{n=1}^{4} \frac{\left(-\frac{i \Delta t}{\hbar} H\right)^{n}}{n !} \psi_{k}(\mathbf{r}, t) .
$$

The choice of the total propagation time of about $30 \mathrm{fs}$ is dictated by the fact that it must well exceed a typical stretching period. For a $\mathrm{Si}-\mathrm{H}$ bond this stretching period is roughly around 8 fs.

To represent Kohn-Sham orbitals and the density of electrons we used numerical grids defined within a sufficiently large simulation box. The use of numerical grids has certain merits in the calculations such as those carried out in the present work. Since the oscillations of the strong laser electric field may result in a significant movement of the electron density, one must be able to accurately represent the Kohn-Sham orbitals not only around the ionic centers but also in the regions away from them. Generally, it may not be known a priori where and how the density moves. The use of the numerical grids (in a sufficiently large box) easily addresses this problem. Another merit of the numerical representation of the orbitals is an easy and straightforward control over the accuracy. By adjusting a single parameter, the grid spacing, one may increase or decrease the accuracy of the calculations as needed. Lastly, the implementation of the time development algorithms is greatly simplified when numerical grids are employed.

In the present work, we used uniform numerical grids with the $x$-, $y$-, and $z$-spacing of $0.25 \AA$. The distance from the leftmost (rightmost) ion to the edge of the simulation box was $4.8 \AA$ or larger. Due to the polarization of the laser, the electron density oscillates mainly along the $x$-axis. The motion of the desorbed (or oscillating) ions also takes place along the $x$-axis. For this reason, the minimal distance from the leftmost (rightmost) ion to the edge of the box along the $x$-axis was extended to at least $7.7 \AA$.

Set of Eqs. (1) describes the time evolution of the electrons in the system. The dynamics of the ions in our simulations was treated classically within the Ehrenfest scheme. The ions were allowed to move classically under the influence of quantum forces computed as the derivatives of the total energy with respect to ionic positions:

$$
\begin{aligned}
M_{i} \frac{d^{2} \mathbf{R}_{i}}{d t^{2}}= & -\nabla_{\mathbf{R}_{i}}\left[Z_{i} V^{\text {ext }}\left(\mathbf{R}_{i}, t\right)\right. \\
& \left.+\sum_{j \neq i}^{N_{\text {ions }}} \frac{Z_{i} Z_{j}}{\left|\mathbf{R}_{i}-\mathbf{R}_{j}\right|}+\int V^{\text {ion }}\left(\mathbf{r}, \mathbf{R}_{i}\right) \rho(\mathbf{r}, t) d \mathbf{r}\right]
\end{aligned}
$$

Here $M_{i}$ is the mass of the $i$-th ion and $Z_{i}$ is its pseudocharge (valence). Newton's equations (8) are coupled with the timedependent Kohn-Sham equations (1) through the electron density $\rho$, and thus the entire system of equations has to be solved simultaneously as the time evolves. We would like to note that application of Eq. (8) requires the validity of the Hellmann-Feynman theorem for time-dependent wave functions/orbitals. Strictly speaking, this is not the case should any motion of nuclei take place. Therefore, the Ehrenfest scheme as such is approximate. Methods that go beyond the classical Ehrenfest dynamics, in particular the trajectory hopping methods, have been an area of active research. ${ }^{79-90}$ However, at present time, the applicability of these methods to large systems remains limited due to increased complexity and computational demands.

As we used finite surface fragments, only certain ions were allowed to move in our simulations. The rest were fixed in order to preserve the general geometric structure of the surface. To assess the reliability and convergence of the results and to detect possible artifacts originating from the finite size of the structures all simulations were performed for two surface fragments of different size: $\mathrm{Si}_{22} \mathrm{H}_{28}$ and $\mathrm{Si}_{46} \mathrm{H}_{46}$. The corresponding structures are shown in Fig. 1. Both fragments are four-layer silicon structures with $100 \%$ hydrogen coverage on the surface. Additionally, we used hydrogens to passivate all dangling silicon bonds on all other sides of the structures. The bond lengths were taken from Ref. 19, where relaxations of the $\mathrm{H}-\mathrm{Si}(111)$ surface were determined using DFT calculations in a slab configuration. Specifically, the bond lengths between neighboring ions were as follows: $1.54 \AA$ (H-Si1), $2.331 \AA$ (Si1-Si2), $2.320 \AA$ (Si2-Si3), and $2.352 \AA$ (Si3-Si4). The latter corresponds to the structure of bulk silicon. Here $\mathrm{Si} 1, \mathrm{Si} 2, \mathrm{Si} 3$, and $\mathrm{Si} 4$ denote the silicon ions in layers $1,2,3$, and 4, respectively. The hydrogen ions used to passivate dangling bonds were placed $1.48 \AA$ from the corresponding silicon ions. The above bond lengths are in agreement with those computed in Ref. 91. While according to Ref. 19 the Si-layers near the surface are slightly more compressed, we believe that such fine details would not have any qualitative effect on the results of our simulations.

Apparently, hydrogen desorption from $\mathrm{Si}$ surface can only occur when a significant absorption of the energy of the laser electric field takes place. This absorption is wavelength
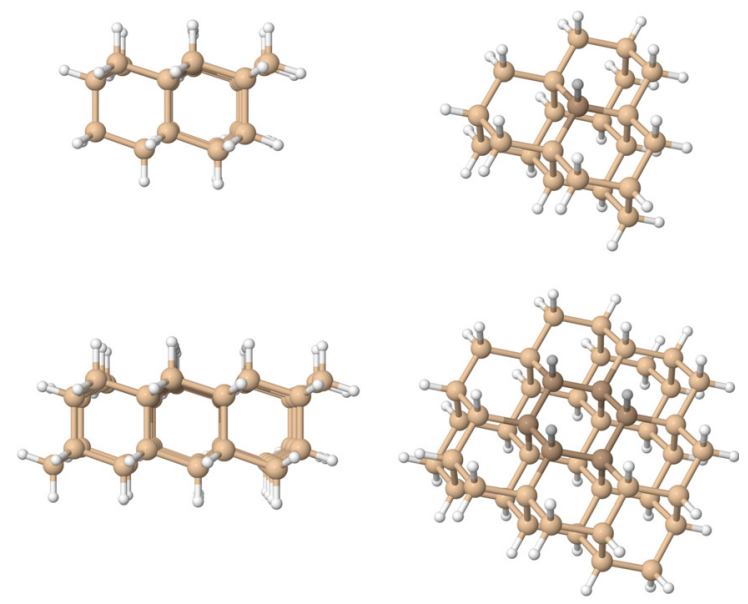

FIG. 1. (Color online) Two fragments of the H-Si(111) surface used in our calculations: $\mathrm{Si}_{22} \mathrm{H}_{28}$ (top) and $\mathrm{Si}_{46} \mathrm{H}_{46}$ (bottom). The ions which were allowed to move are indicated with dark color. 
dependent. In order to have a rough estimate which laser wavelength may be suitable in the low laser intensity regime, one can perform the calculation of the optical absorption spectra. In the TDDFT framework they can be obtained by perturbing the electrons in the system with a weak $\delta$-kick and monitoring the evolution of the dipole moment with time. ${ }^{41,92}$ The $\delta$-shape of the perturbing potential provides equal weights to all excitation frequencies $\omega$. The action of the $\delta$-kick amounts to multiplying the Kohn-Sham orbitals with the following exponential factor at the time $t=0$ :

$$
\psi_{k}\left(\mathbf{r}, t=0^{+}\right)=e^{i p x} \psi_{k}(\mathbf{r}, t=0), \quad p \ll L^{-1},
$$

where $L$ is the linear size of the system. The kick creates small oscillations of the dipole moment, $d(t)$, which can be recorded for some sufficiently long time. Then $d(t)$ can be Fourier-transformed, giving the dynamic polarizability of the system,

$$
\alpha(\omega)=\frac{1}{p} \int[d(t)-d(0)] e^{i \omega t} f(t) \mathrm{d} t
$$

Here $f(t)$ is some damping factor insuring the existence of the Fourier transform. Lastly, the photoabsorption cross section is related to the dynamic polarizability as

$$
\sigma(\omega)=\frac{4 \pi \omega}{c} \Im[\alpha(\omega)]
$$

where $c$ is the speed of light and $\Im$ stands for the imaginary part. It should be noted that the way the optical absorption spectra are calculated assumes the weakness of the perturbing electric field. Thus, in the regime when the laser field is no longer weak compared to the typical field strengths experienced by valence electrons in an atom, one may expect drastic changes in the photoabsorption properties.

\section{RESULTS}

A schematic drawing of the computational setup for our simulations is shown in Fig. 2. In this setup, a system under study is placed in a simulation box and a laser field with the polarization along the $x$-axis is applied. In those cases when the desorption of atoms takes place they move away from the surface in the $x$-direction as well.

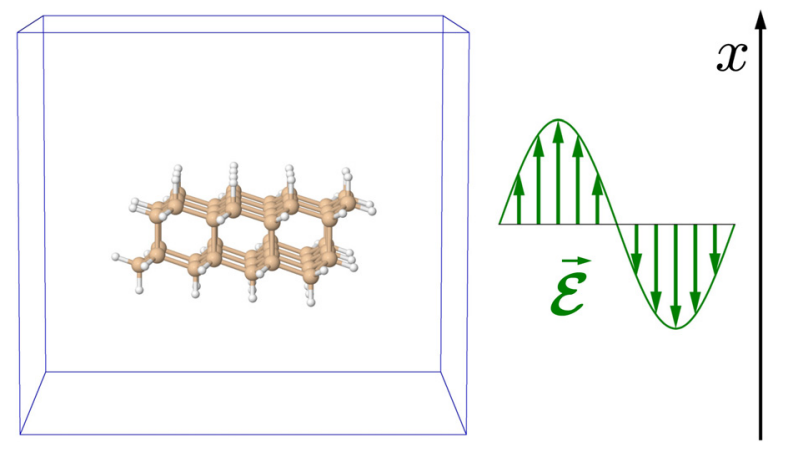

FIG. 2. (Color online) Computational set-up: surface fragment in a simulation box with oscillating electric field in $x$-direction.
Before carrying out the simulations with the laser field we have computed the optical absorption spectra for the surface fragments by time-propagating the Kohn-Sham orbitals for $20 \mathrm{fs}$ and using relations (9)-(11). The results given in Fig. 3 show that there is a significant optical gap of approximately $5 \mathrm{eV}$ for both the $\mathrm{Si}_{22} \mathrm{H}_{28}$ and $\mathrm{Si}_{46} \mathrm{H}_{46}$ fragments. They suggest that very little or no energy absorption should take place below the frequency corresponding to $5-7 \mathrm{eV}$ if a low intensity laser is applied.

The maximum amplitude of the laser field, $\mathcal{E}_{\max }$, used in our simulations ranged from $0.5 \mathrm{~V} / \AA$ to $4.0 \mathrm{~V} / \AA$, which corresponds to intensities of $3.3 \times 10^{16}$ and $2.1 \times 10^{18} \mathrm{~W} / \mathrm{m}^{2}$, respectively. After 25 fs the corresponding pulse with envelope (7) yields the fluence value ranging from $6.7 \mathrm{~J} / \mathrm{m}^{2}$ to $3.4 \times 10^{3} \mathrm{~J} / \mathrm{m}^{2}$. Such intensities should generally be considered high. For comparison, in the hydrogen atom, at $r_{\text {Bohr }}=0.53 \AA$ the electron experiences a field of $51 \mathrm{~V} / \AA$ due to the proton; at the same time the duration of our laser pulse exceeds the time scale of the electronic motion $(\sim 0.2 \mathrm{fs})$ by two orders of magnitude. We used three different laser wavelengths in our calculations: 99,124 , and $248 \mathrm{~nm}$, which correspond to 5,10 , and $12.5 \mathrm{eV}$ photon energy. This choice of the wavelengths is not of particular importance. Other values in the UV range could have been used as well since the nature of the processes we simulated is not resonant. Our choice was based on the absorption spectra shown in Fig. 3. We picked one photon energy that is lower than the optical gap, while the other two photon energies lie in the range where the absorption strength is expected to be high (even at low intensities of the laser).

As was mentioned in Sec. II, only certain ions were allowed to move freely in our simulations. In the case of $\mathrm{Si}_{22} \mathrm{H}_{28}$ fragment there were only two such ions: a hydrogen ion at the center of the surface and the silicon atom that has a chemical bond with the hydrogen. The positions of other ions were fixed. For the $\mathrm{Si}_{46} \mathrm{H}_{46}$ fragment, out of 92 ions in the system, 9 were allowed to move: three $\mathrm{H}$ ions (those that are closest to the surface center), three $\mathrm{Si}$ ions in the first atomic plane, and three $\mathrm{Si}$ ions in the second atomic plane. All these ions are marked with dark color in Fig. 1). The simulations for both fragments were in good qualitative agreement as to when and how the desorption of the hydrogen

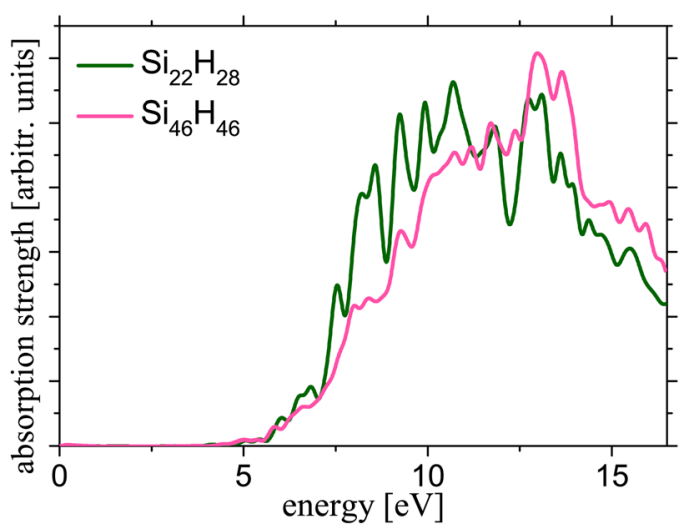

FIG. 3. (Color online) Absorption spectra calculated using the linear response model. 

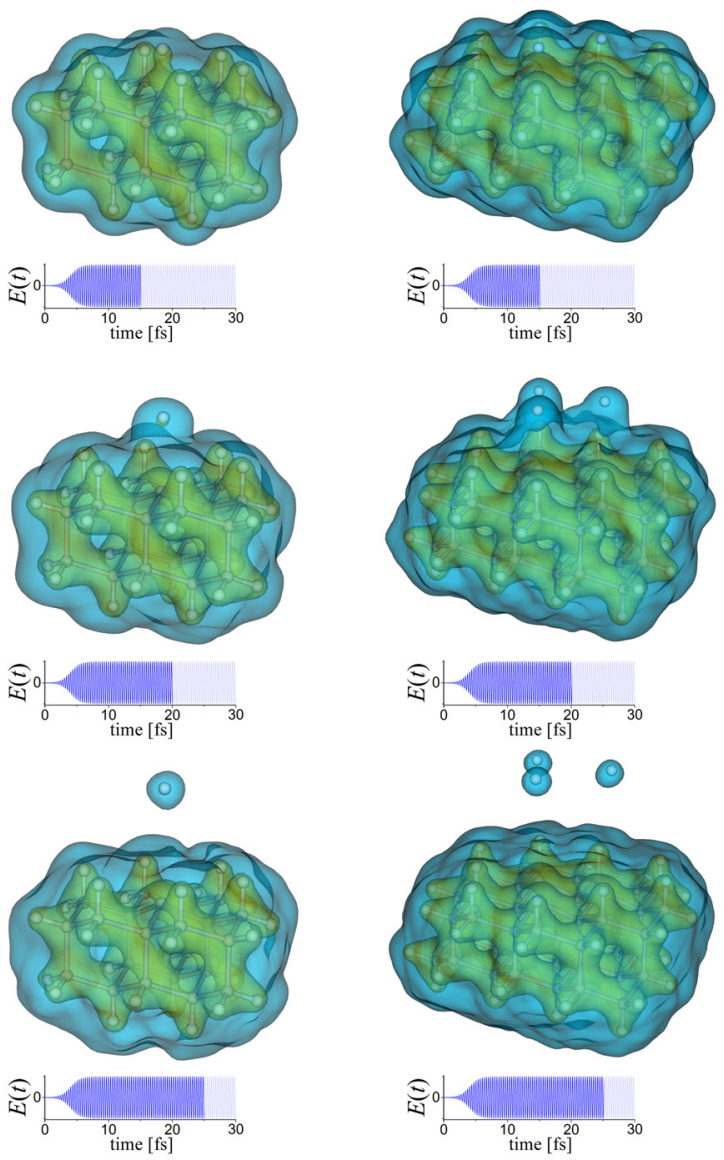

FIG. 4. (Color online) Time-evolution of the electron density and ion dynamics in $\mathrm{Si}_{22} \mathrm{H}_{28}$ (left column) and $\mathrm{Si}_{46} \mathrm{H}_{46}$ (right column) surface fragments for the case of $\mathcal{E}_{\max }=1.0 \mathrm{~V} / \AA$ and $99 \mathrm{~nm}$ laser wavelength. Two isosurfaces corresponding to the electron density of 0.15 and $0.015 \AA^{-3}$ are plotted at $t=0,15$, 20, and 25 fs (enhanced online) [URL: http://dx.doi.org/10.1063/1.3638064.1]. occurs, indicating that our finite-fragment model of the H$\mathrm{Si}(111)$ surface is adequate for the type of calculations we have performed.

The qualitative picture of the simulated process is as follows. Upon the arrival of the laser pulse, the electron density starts to oscillate in the direction perpendicular to the surface. This oscillation of the density creates time-dependent forces on ions and a slow nuclear motion begins. In particular, those ions close to the surface experience a non-zero time-averaged force. The hydrogen ions are lighter than the silicon ones. Due to the force and a small mass they respond to the laser more quickly and, if the intensity of the laser is sufficiently high, the $\mathrm{Si}-\mathrm{H}$ bond breaks and the hydrogens eventually fly away from the surface. A few snapshots of this process of bond scission are presented in Fig. 4.

A more quantitative picture of hydrogen desorption can be devised from the plots of ionic positions, which we present in Fig. 5. In this figure we show the positions of the hydrogen and the neighboring silicon atom in the case of a $\mathrm{Si}_{22} \mathrm{H}_{28}$ fragment subjected to a laser field of different amplitudes and frequencies. As can be seen from the figure, for the smallest laser amplitude of $0.5 \mathrm{~V} / \AA$ the dissociation does not occur during the first 25 femtoseconds of the simulation. The position of the hydrogen does change with time, yet the effect is not strong enough to rip the ion off the surface completely. If a laser pulse with such an amplitude is discontinued and the deposition of energy is stopped then the electronic and vibrational excitation energy accumulated in the system would eventually get redistributed in the (infinite) lattice. When the amplitude of the laser electric field is increased to $1.0 \mathrm{~V} / \AA$, the hydrogen gets desorbed in the case of shorter wavelengths ( 99 and $124 \mathrm{~nm}$ ) and stays at the surface in the case of the $248 \mathrm{~nm}$ wavelength. As far as the
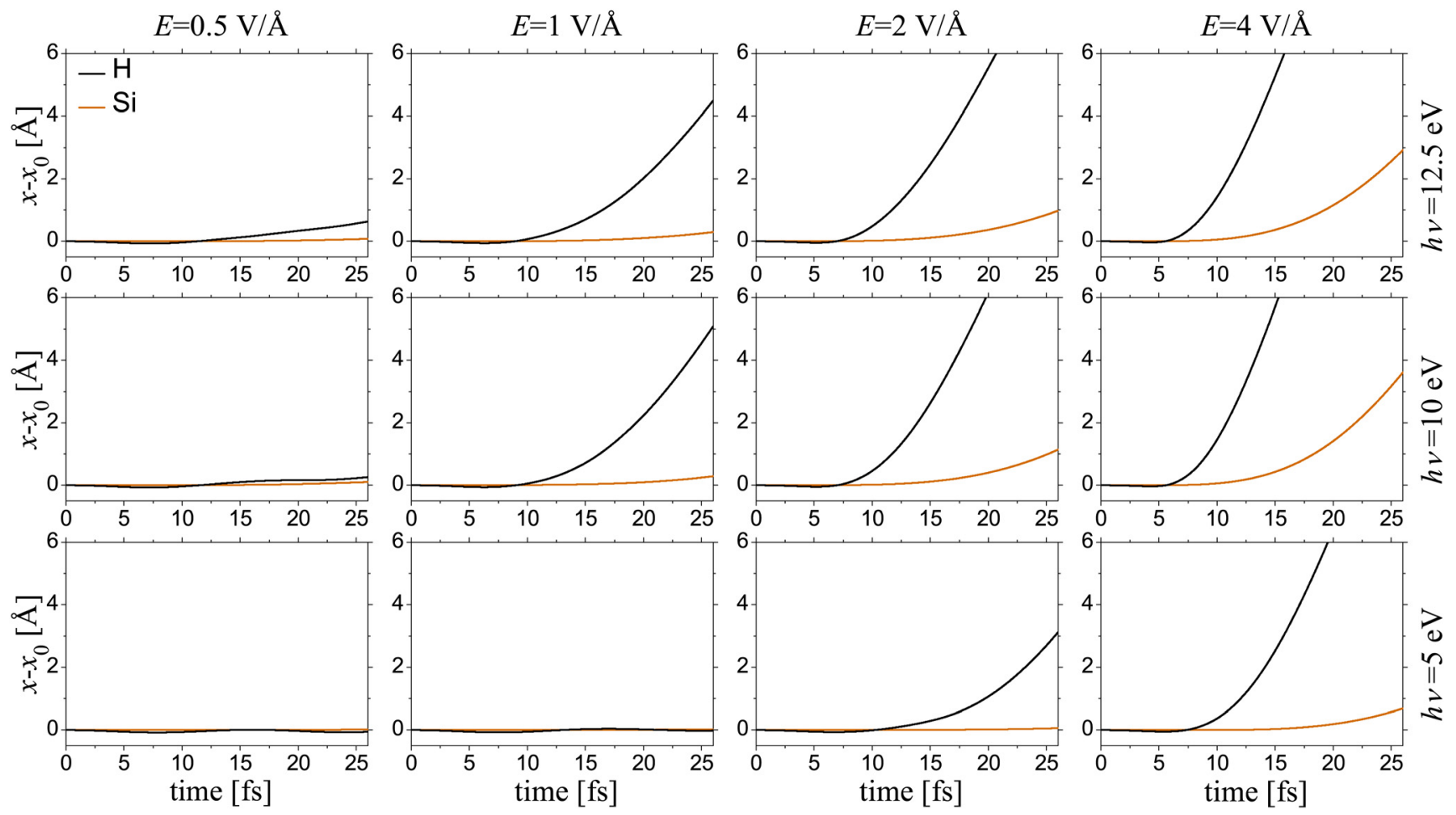

FIG. 5. (Color online) Time-evolution of the $x$-displacement of $\mathrm{H}$ and $\mathrm{Si}$ ions for different laser electric field intensities and frequencies in the case of a $\mathrm{Si}_{22} \mathrm{H}_{28}$ fragment. 
silicon ion is concerned, its $x$-displacement remains small regardless of the wavelength. Hence, the results of our simulations suggest that short (tens of femtoseconds) laser pulses of UV light can be used to strip off hydrogens without changing the structure of the underlying silicon. For this to occur one has to choose appropriate values of the laser wavelength, intensity, and pulse duration. Experimental observation of desorption of atomic hydrogen from Si surfaces using 157 and $308 \mathrm{~nm}$ lasers has been reported in Refs. 9 and 93. It should also be noted that in the absence of hydrogen coverage, the $\mathrm{Si}(111)$ surface in vacuum eventually evolves into a different reconstruction. Modeling this process, however, lies outside of the scope of the present work.

When the amplitude of the applied laser field is increased to $2.0 \mathrm{~V} / \AA$ the dissociation of hydrogen occurs even for $5.0 \mathrm{eV}$ photon energy, which clearly indicates the onset of the nonlinear regime. Also, in the case of 10 and $12.5 \mathrm{eV}$ photon energies, the silicon ion begins to acquire a significant momentum. The magnitude of the $x$-displacement of the silicon ion becomes even larger at $\mathcal{E}_{\max }=4.0 \mathrm{~V} / \AA$, which is the largest laser amplitude we used in this work. In the latter case, the top silicon atoms on the H-Si(111) surface break their bonds with other neighboring $\mathrm{Si}$ atoms and escape. This is in agreement with the experimental results of Ref. 15 where laser induced desorption of $\mathrm{Si}$ atoms from a clean Si surface has been observed.

We repeated all our simulations for a $\mathrm{Si}_{46} \mathrm{H}_{46}$ fragment and found very good qualitative agreement with the case of $\mathrm{Si}_{22} \mathrm{H}_{28}$, which can be seen in Fig. 6. As far as the motion of the hydrogen ions and the silicon ions from the first Si-plane is concerned, they followed $x$-trajectories that are very close to those of the $\mathrm{H}$ and $\mathrm{Si}$ ions in $\mathrm{Si}_{22} \mathrm{H}_{28}$. In the larger fragment we had non-fixed silicon ions from the second Si-plane. We found their $x$-displacements to be significantly smaller than those of the silicon ions in the first Si-plane. For the case of $\mathcal{E}_{\max }=4.0 \mathrm{~V} / \AA$ and $\lambda=99$ and $124 \mathrm{~nm}$, when the displacement of all moving ions was largest, at the end of the simulations the $\mathrm{Si} 2$ ions were approximately twice closer to their initial positions compared to the $\mathrm{Si} 2$ ions. The good agreement between the results obtained for $\mathrm{Si}_{22} \mathrm{H}_{28}$ and $\mathrm{Si}_{46} \mathrm{H}_{46}$ surface fragments indicates that the simulation results can be meaningfully extrapolated to the case of an infinite $\mathrm{H}-\mathrm{Si}(111)$ surface.

The motion of all ions in our simulations was completely independent and not subjected to any constraints aiming to preserve the $C_{3 v}$ symmetry, which both fragments should possess if the electric field is uniform in space at any given instant and is directed along the $\mathrm{x}$ axis. Our computational model, however, employs uniform rectangular grids (obviously not of $C_{3 v}$ symmetry). Despite this fact the system maintained the $C_{3 v}$ symmetry by itself to a very high degree, which serves as an additional check as to whether the choice of the simulation parameters (box size, grid spacing, etc.) was adequate.

Lastly, we have studied how isotopic substitution of hydrogen ions in $\mathrm{H}-\mathrm{Si}(111)$ affects the outcome of our simulations. We have repeated several calculations with the $\mathrm{Si}_{22} \mathrm{D}_{28}$ fragment where the mass of the moving hydrogen ion was set to be equal to the mass of the deuterium and ${ }^{28} \mathrm{H}$ (hydrogen with the mass of silicon). The results of these calculations are presented in Fig. 7. The $x$-displacement plots in this figure suggest that the higher mobility of the hydrogen ions is due to their light mass. Upon increasing the nuclear mass, the motion of $\mathrm{H}$ ions becomes less responsive and in
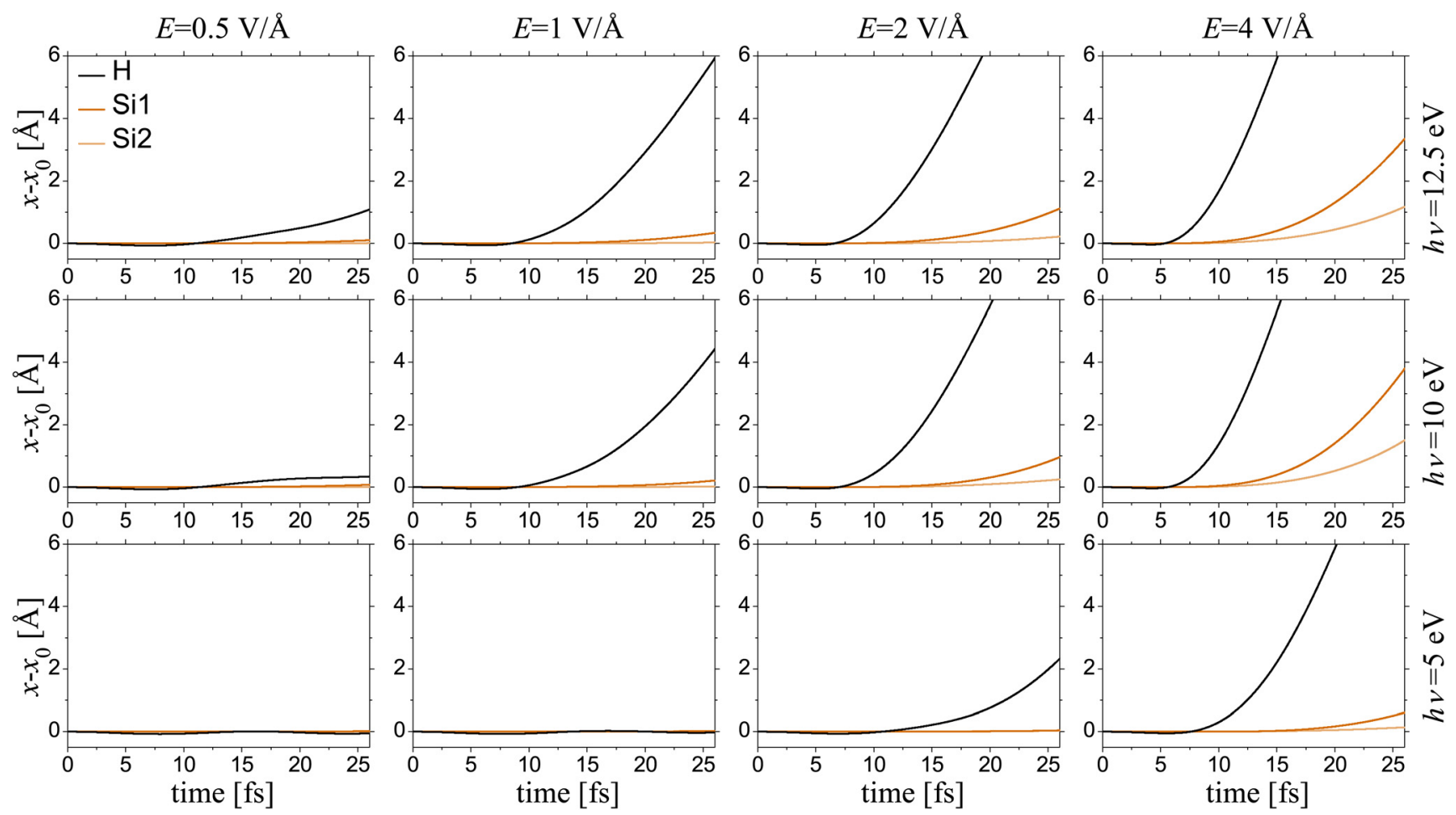

FIG. 6. (Color online) Time-evolution of the $x$-displacement of $\mathrm{H}$ and $\mathrm{Si}$ ions for different laser electric field intensities and frequencies in the case of a $\mathrm{Si}_{46} \mathrm{H}_{46}$ fragment. Si1 and $\mathrm{Si} 2$ denote ions in atomic plane 1 and 2, respectively. Si1 is the closest one to the H-plane. 

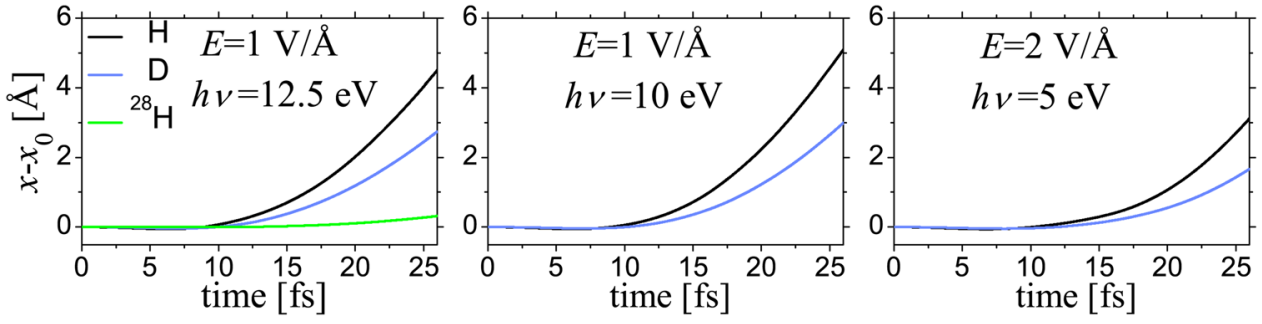

FIG. 7. (Color online) Decrease in the magnitude of the $x$-displacement as a result of isotopic substitution $\mathrm{H} \rightarrow \mathrm{D} \rightarrow$ ${ }^{28} \mathrm{H}$ in a $\mathrm{Si}_{22} \mathrm{H}_{28}$ fragment. the case of ${ }^{28} \mathrm{H}$ they are essentially as slow as the silicon ions next to the surface.

One possible interpretation of the mechanism of the force acting on ions can be related to emission of electrons from the surface. Due to a large magnitude of the laser field, a certain degree of the surface ionization takes place. In the limiting case of even stronger field than used in our simulations, the finite surface fragment as a whole would undergo an ionization followed by a Coulomb explosion. In our computational setup with relatively large, but still finite simulation box it is hard to quantify precisely how many electrons left the surface completely. This is because the electron density is quite distorted compared to the ground state density and oscillates up and down. However, rough estimates in the case of 2 and $4 \mathrm{~V} / \AA$ laser amplitude suggest that a few electrons (out of 230 for $\mathrm{Si}_{46} \mathrm{H}_{46}$ ) should probably be considered escaped by the end of the simulation.

To conclude, the dynamics of laser induced bond breaking at a hydrogenated silicon surface has been studied computationally in the framework of TDDFT. The calculations demonstrate that the laser assisted desorption of the hydrogen layer should be possible. At the same time the process exhibits highly nonlinear behavior. Nonetheless, by choosing appropriate laser parameters the hydrogen layer can be removed without destroying the Si surface.

\section{ACKNOWLEDGMENTS}

This work has been supported by National Science Foundation through Grant No. CMMI-0927345. The authors also thank Joseph A. Driscoll for discussions and suggestions concerning the manuscript.

${ }^{1}$ A. Fuhrer, M. Füchsle, T. C. G. Reusch, B. Weber, and M. Y. Simmons, Nano Lett. 9, 707 (2009).

${ }^{2}$ F. J. Ruess, L. Oberbeck, M. Y. Simmons, K. E. J. Goh, A. R. Hamilton, T. Hallam, S. R. Schofield, N. J. Curson, and R. G. Clark, Nano Lett. 4, 1969 (2004).

${ }^{3}$ S. R. Schofield, N. J. Curson, M. Y. Simmons, F. J. Rue, T. Hallam, L. Oberbeck, and R. G. Clark, Phys. Rev. Lett. 91, 136104 (2003).

${ }^{4}$ J. J. Boland, Phys. Rev. Lett. 67, 1539 (1991).

${ }^{5}$ T. Hallam, T. C. G. Reusch, L. Oberbeck, N. J. Curson, and M. Y. Simmons, J. Appl. Phys. 101, 034305 (2007).

${ }^{6}$ R. S. Becker, G. S. Higashi, Y. J. Chabal, and A. J. Becker, Phys. Rev. Lett. 65, 1917 (1990)

${ }^{7}$ T.-C. Shen, C. Wang, G. C. Abeln, J. R. Tucker, J. W. Lyding, P. Avouris, and R. E. Walkup, Science 268, 1590 (1995).

${ }^{8}$ M. Dürr, Z. Hu, A. Biedermann, U. Höfer, and T. F. Heinz, Phys. Rev. Lett. 88, 046104 (2002)

${ }^{9}$ T. Vondrak and X.-Y. Zhu, Phys. Rev. Lett. 82, 1967 (1999).

${ }^{10}$ A. Assion, T. Baumert, M. Bergt, T. Brixner, B. Kiefer, V. Seyfried, M. Strehle, and G. Gerber, Science 282, 919 (1998).

${ }^{11}$ R. J. Levis, G. M. Menkir, and H. Rabitz, Science 292, 709 (2001).
${ }^{12}$ C. Daniel, J. Full, L. González, C. Lupulescu, J. Manz, A. Merli, S. Vajda, and L. Wöste, Science 299, 536 (2003).

${ }^{13}$ Z. Liu, L. C. Feldman, N. H. Tolk, Z. Zhang, and P. I. Cohen, Science 312, 1024 (2006).

${ }^{14}$ M. Müllenborn, K. Birkelund, F. Grey, and S. Madsen, Appl. Phys. Lett. 69, 3013 (1996).

${ }^{15}$ J. Kanasaki, K. Iwata, and K. Tanimura, Phys. Rev. Lett. 82, 644 (1999).

${ }^{16}$ K. Bobrov, G. Comtet, G. Dujardin, and L. Hellner, Phys. Rev. Lett. 86, 2633 (2001).

${ }^{17}$ P. Avouris, R. E. Walkup, A. R. Rossi, T. C. Shen, G. C. Abeln, J. R. Tucker, and J. W. Lyding, Chem. Phys. Lett. 257, 148 (1996).

${ }^{18}$ P. Guyot-Sionnest, P. Dumas, Y. J. Chabal, and G. S. Higashi, Phys. Rev. Lett. 64, 2156 (1990).

${ }^{19}$ E. Kaxiras and J. D. Joannopoulos, Phys. Rev. B 37, 8842 (1988).

${ }^{20}$ K. J. Chang and D. J. Chadi, Phys. Rev. B 42, 7651 (1990).

${ }^{21}$ R. Stumpf and P. M. Marcus, Phys. Rev. B 47, 16016 (1993).

${ }^{22}$ H. Gai and G. A. Voth, J. Chem. Phys. 101, 1734 (1994).

${ }^{23}$ B. Tuttle and C. G. Van de Walle, Phys. Rev. B 59, 12884 (1999).

${ }^{24}$ H. Lim, K. Cho, I. Park, J. D. Joannopoulos, and E. Kaxiras, Phys. Rev. B 52, 17231 (1995).

${ }^{25}$ X.-P. Li and D. Vanderbilt, Phys. Rev. Lett. 69, 2543 (1992).

${ }^{26}$ G. Boendgen and P. Saalfrank, J. Phys. Chem. B 102, 8029 (1998).

${ }^{27}$ C. J. Wu, I. V. Ionova, and E. A. Carter, Surf. Sci. 295, 64 (1993).

${ }^{28}$ B. Koehler and S. George, Surf. Sci. 248, 158 (1991).

${ }^{29}$ K. W. Kolasinski, Curr. Opin. Solid State Mater. Sci. 8, 353 (2004).

${ }^{30}$ P. J. Hay, R. C. Boehm, J. D. Kress, and R. L. Martin, Surf. Sci. 436, 175 (1999).

${ }^{31}$ J. Ma, E. Wang, Z. Zhang, and B. Wu, Phys. Rev. B 78, 125303 (2008).

${ }^{32}$ K. W. Kolasinski, W. Nessler, A. de Meijere, and E. Hasselbrink, Phys. Rev. Lett. 72, 1356 (1994).

${ }^{33}$ M. F. Hilf and W. Brenig, J. Chem. Phys. 112, 3113 (2000).

${ }^{34}$ M. R. Radeke and E. A. Carter, Phys. Rev. B 55, 4649 (1997).

${ }^{35}$ Y. Miyamoto and O. Sugino, Phys. Rev. B 62, 2039 (2000).

${ }^{36}$ S. Bubin and K. Varga, Appl. Phys. Lett. 98, 154101 (2011).

${ }^{37}$ P. Hohenberg and W. Kohn, Phys. Rev. 136, B864 (1964).

${ }^{38}$ W. Kohn and L. J. Sham, Phys. Rev. 140, A1133 (1965).

${ }^{39}$ E. Runge and E. Gross, Phys. Rev. Lett. 52, 997 (1984).

${ }^{40}$ K. Burke, J. Werschnik, and E. K. U. Gross, J. Chem. Phys. 123, 062206 (2005).

${ }^{41}$ K. Yabana and G. F. Bertsch, Phys. Rev. B 54, 4484 (1996).

${ }^{42}$ M. A. L. Marques, A. Castro, G. F. Bertsch, and A. Rubio, Comput. Phys. Commun. 151, 60 (2003)

${ }^{43}$ A. Tsolakidis, D. Sánchez-Portal, and R. M. Martin, Phys. Rev. B 66, 235416 (2002).

${ }^{44}$ A. Castro, M. A. L. Marques, and A. Rubio, J. Chem. Phys. 121, 3425 (2004).

${ }^{45}$ R. Bauernschmitt and R. Ahlrichs, Chem. Phys. Lett. 256, 454 (1996).

${ }^{46}$ M. E. Casida, C. Jamorski, K. C. Casida, and D. R. Salahub, J. Chem. Phys. 108, 4439 (1998).

${ }^{47}$ M. Lopez del Puerto, M. L. Tiago, and J. R. Chelikowsky, Phys. Rev. B 77, 045404 (2008).

${ }^{48}$ S. Meng and E. Kaxiras, J. Chem. Phys. 129, 054110 (2008).

${ }^{49}$ M. Valiev, E. Bylaska, N. Govind, K. Kowalski, T. Straatsma, H. V. Dam, D. Wang, J. Nieplocha, E. Apra, T. Windus, and W. de Jong, Comput. Phys. Commun. 181, 1477 (2010).

${ }^{50}$ X. Gonze, B. Amadon, P.-M. Anglade, J.-M. Beuken, F. Bottin, P. Boulanger, F. Bruneval, D. Caliste, R. Caracas, M. Côté, T. Deutsch, L. Genovese, P. Ghosez, M. Giantomassi, S. Goedecker, D. Hamann, P. Hermet, F. Jollet, G. Jomard, S. Leroux, M. Mancini, S. Mazevet, M. Oliveira, G. Onida, Y. Pouillon, T. Rangel, G.-M. Rignanese, D. Sangalli, R. Shaltaf, M. Torrent, M. Verstraete, G. Zerah, and J. Zwanziger, Comput. Phys. Commun. 180, 2582 (2009). 
${ }^{51}$ A. Marini, C. Hogan, M. Grüning, and D. Varsano, Comput. Phys. Commun. 180, 1392 (2009).

${ }^{52}$ K. Yabana and G. F. Bertsch, Phys. Rev. A 60, 1271 (1999).

${ }^{53}$ W. R. Burdick, Y. Saad, L. Kronik, I. Vasiliev, M. Jain, and J. R. Chelikowsky, Comput. Phys. Commun. 156, 22 (2003).

${ }^{54}$ H. Tal-Ezer and R. Kosloff, J. Chem. Phys. 81, 3967 (1984).

${ }^{55}$ D. Kosloff and R. Kosloff, J. Comput. Phys. 52, 35 (1983).

${ }^{56}$ S. A. Chin and C. R. Chen, J. Chem. Phys. 114, 7338 (2001).

${ }^{57}$ X. Zhou and C. D. Lin, Phys. Rev. A 61, 053411 (2000).

${ }^{58}$ I. Yabana, T. Nakatsukasa, and G. F. Bertsch, Phys. Status Solidi B 243, 1121 (2006).

${ }^{59}$ D. Rocca, R. Gebauer, Y. Saad, and S. Baroni, J. Chem. Phys. 128, 154105 (2008).

${ }^{60}$ J. Crank and P. Nicolson, Math. Proc. Cambridge Philos. Soc. 43, 50 (1947).

${ }^{61}$ X. Chu and S.-I. Chu, Phys. Rev. A 64, 063404 (2001).

${ }^{62}$ Y. Kawashita, T. Nakatsukasa, and K. Yabana, J. Phys.: Condens. Matter 21, 064222 (2009).

${ }^{63}$ J.-I. Iwata, K. Yabana, and G. F. Bertsch, J. Chem. Phys. 115, 8773 (2001).

${ }^{64}$ T. Nakatsukasa and K. Yabana, J. Chem. Phys. 114, 2550 (2001).

${ }^{65}$ M. J. Stott and E. Zaremba, Phys. Rev. A 21, 12 (1980).

${ }^{66}$ F. Kootstra, P. L. de Boeij, and J. G. Snijders, J. Chem. Phys. 112, 6517 (2000).

${ }^{67}$ G. F. Bertsch, J.-I. Iwata, A. Rubio, and K. Yabana, Phys. Rev. B 62, 7998 (2000).

${ }^{68}$ S. J. A. van Gisbergen, J. G. Snijders, and E. J. Baerends, J. Chem. Phys. 109, 10657 (1998).

${ }^{69}$ L. Frediani, Z. Rinkevicius, and H. Ågren, J. Chem. Phys. 122, 244104 (2005).

${ }^{70}$ Y. Takimoto, F. D. Vila, and J. J. Rehr, J. Chem. Phys. 127, 154114 (2007).
${ }^{71}$ A. Görling, Phys. Rev. A 54, 3912 (1996).

${ }^{72}$ N. L. Doltsinis and M. Sprik, Chem. Phys. Lett. 330, 563 (2000).

${ }^{73}$ C. Adamo, G. E. Scuseria, and V. Barone, J. Chem. Phys. 111, 2889 (1999).

${ }^{74}$ S. Kurth, G. Stefanucci, C.-O. Almbladh, A. Rubio, and E. K. U. Gross, Phys. Rev. B 72, 035308 (2005).

${ }^{75}$ X. Qian, J. Li, X. Lin, and S. Yip, Phys. Rev. B 73, 035408 (2006).

${ }^{76}$ A. Marini, R. Del Sole, and A. Rubio, Phys. Rev. Lett. 91, 256402 (2003).

${ }^{77}$ J. P. Perdew and A. Zunger, Phys. Rev. B 23, 5048 (1981).

${ }^{78}$ N. Troullier and J. L. Martins, Phys. Rev. B 43, 1993 (1991).

${ }^{79}$ J. C. Tully and R. K. Preston, J. Chem. Phys. 55, 562 (1971).

${ }^{80}$ J. C. Tully, J. Chem. Phys. 93, 1061 (1990).

${ }^{81}$ T. J. Martinez, M. Ben-Nun, and R. D. Levine, J. Phys. Chem. 100, 7884 (1996).

${ }^{82}$ G. Stock and M. Thoss, Phys. Rev. Lett. 78, 578 (1997).

${ }^{83}$ C. C. Martens and J.-Y. Fang, J. Chem. Phys. 106, 4918 (1997).

${ }^{84}$ O. V. Prezhdo and V. V. Kisil, Phys. Rev. A 56, 162 (1997).

${ }^{85}$ J. C. Tully, Faraday Discuss. 110, 407 (1998).

${ }^{86}$ R. Kapral and G. Ciccotti, J. Chem. Phys. 110, 8919 (1999).

${ }^{87}$ C. F. Craig, W. R. Duncan, and O. V. Prezhdo, Phys. Rev. Lett. 95, 163001 (2005).

${ }^{88}$ E. Tapavicza, I. Tavernelli, and U. Rothlisberger, Phys. Rev. Lett. 98, 023001 (2007).

${ }^{89}$ J. E. Subotnik, J. Chem. Phys. 132, 134112 (2010).

${ }^{90}$ A. Abedi, N. T. Maitra, and E. K. U. Gross, Phys. Rev. Lett. 105, 123002 (2010).

${ }^{91}$ F. Cargnoni, C. Gatti, E. May, and D. Narducci, J. Chem. Phys. 112, 887 (2000).

${ }^{92}$ K. Yabana and G. F. Bertsch, Int. J. Quantum Chem. 75, 55 (1999).

${ }^{93}$ A. Pusel, U. Wetterauer, and P. Hess, Phys. Rev. Lett. 81, 645 (1998). 